\title{
Thyroid Hormone Therapy for Older Adults with Subclinical Hypothyroidism
}

TO THE EDITOR: We would note some limitations in the otherwise informative report by Stott et al. on the Thyroid Hormone Replacement for Untreated Older Adults with Subclinical Hypothyroidism - A Randomized Placebo Controlled Trial (TRUST) (June 29 issue). ${ }^{1}$ The results for free thyroxine were reported only as pretreatment levels at baseline; values after treatment would have been helpful in interpreting the data. Also, lipid levels should have been included in the cardiovascular risk assessment, since hypothyroidism is a known cause of potentially reversible hyperlipidemia. ${ }^{2}$ Patients with hyperlipidemia and subclinical hypothyroidism could well be candidates for a trial of thyroxine supplementation.

Furthermore, the association between subclinical hypothyroidism and heart disease remains unclear. ${ }^{3,4}$ Stott et al. observed an imbalance between the levothyroxine group and the placebo group in the point estimates for cardiovascular deaths and deaths from any cause. This trend, although not statistically significant owing to the small numbers of patients, is nonetheless worrisome. Were the deaths from any cause adjudicated? If so, how many were sudden deaths, and how were those patients represented in the levothyroxine group and the placebo group? Such additional data could be valuable when clinicians are evaluating the potential risks associated with the discretionary supplementation of thyroid hormone in elderly patients with subclinical hypothyroidism.

Amie Ogunsakin, M.D.

Solomon S. Solomon, M.D.

Samuel Dagogo-Jack, M.D.

University of Tennessee Health Science Center

Memphis, TN

sdj@uthsc.edu

No potential conflict of interest relevant to this letter was reported.

1. Stott DJ, Rodondi N, Kearney PM, et al. Thyroid hormone therapy for older adults with subclinical hypothyroidism. N Engl J Med 2017;376:2534-44.
2. Danese MD, Ladenson PW, Meinert CL, Powe NR. Effect of thyroxine therapy on serum lipoproteins in patients with mild thyroid failure: a quantitative review of the literature. J Clin Endocrinol Metab 2000;85:2993-3001.

3. Hak AE, Pols HA, Visser TJ, Drexhage HA, Hofman A, Witteman JC. Subclinical hypothyroidism is an independent risk factor for atherosclerosis and myocardial infarction in elderly women: the Rotterdam Study. Ann Intern Med 2000;132:270-8. 4. Cappola AR, Fried LP, Arnold AM, et al. Thyroid status, cardiovascular risk, and mortality in older adults. JAMA 2006;295: 1033-41.

DOI: 10.1056/NEJMc1709989

TO THE EDITOR: On the basis of previous observational findings, ${ }^{1}$ the TRUST trial was initiated to provide evidence as to whether subclinical hypothyroidism is causally linked to disease in old age. Despite being the largest trial to date with 737 participants, it was underpowered to address the association between hypothyroidism and cardiovascular disease, as highlighted in the Discussion section.

We conducted a two-sample mendelian randomization study with 20 genetic variants for circulating levels of thyrotropin and 3 genetic variants for circulating levels of free thyroxine ${ }^{2}$ on summary data of a genomewide association study consisting of 60,801 case patients with coronary artery disease and 123,504 controls from the CARDIoGRAMplusC4D Consortium. ${ }^{3}$ On the basis of statistical analyses that have been described previously, ${ }^{4}$ we found no evidence for a causal relationship between circulating levels of thyrotropin or free thyroxine and the risk of coronary artery disease (odds ratio of 0.99 per standard deviation of increase in thyrotropin, $\mathrm{P}=0.83$; and odds ratio of 1.02 per standard deviation of increase in free thyroxine, $\mathrm{P}=0.74)$. Thus, even if the TRUST trial had been considerably larger and had longer follow-up, it is likely that the investigators would have found no beneficial effect of levothyroxine treatment on the incidence of coronary artery disease, similar to their findings with respect to other outcomes. 
Nicolien A. van Vliet, M.D.

Raymond Noordam, Ph.D.

Diana van Heemst, Ph.D.

Leiden University Medical Center

Leiden, the Netherlands

d.van_heemst@lumc.nl

No potential conflict of interest relevant to this letter was reported.

1. Rodondi N, den Elzen WP, Bauer DC, et al. Subclinical hypothyroidism and the risk of coronary heart disease and mortality. JAMA 2010;304:1365-74.

2. Porcu E, Medici M, Pistis G, et al. A meta-analysis of thyroidrelated traits reveals novel loci and gender-specific differences in the regulation of thyroid function. PLoS Genet 2013;9(2):e1003266. 3. Nikpay M, Goel A, Won $\mathrm{HH}$, et al. A comprehensive 1,000 Genomes-based genome-wide association meta-analysis of coronary artery disease. Nat Genet 2015;47:1121-30.

4. Bos MM, Smit RAJ, Trompet S, van Heemst D, Noordam R Thyroid signaling, insulin resistance, and 2 diabetes mellitus: a mendelian randomization study. J Clin Endocrinol Metab 2017; 102:1960-70.

DOI: 10.1056/NEJMc1709989

TO THE EDITOR: Subclinical hypothyroidism is a common biochemical diagnosis, defined as increased levels of serum thyrotropin with normal levels of serum free thyroxine. The use of the commonly employed direct immunoassay to measure thyrotropin and free thyroxine has some major limitations. ${ }^{1}$ Such limitations are particularly due to changes in binding protein concentrations that occur in many physiologic and disease states and a weak inverse linear log relationship to thyrotropin in thyroid disorders, factors that frequently do not fit in with the clinical presentation. ${ }^{2}$ The measurements of free thyroxine and free triiodothyronine followed by liquid chromatography-mass spectrometry are the most clinically relevant for the evaluation of thyroid disorders and can be achieved with the use of equilibrium dialysis or ultrafiltration to measure the free thyroid concentrations. ${ }^{3}$ In one study, approximately two thirds of the patients with subclinical hypothyroidism (as ascertained by means of immunoassays) were found to have true hypothyroidism when these definitive mass spectrometric methods were used. ${ }^{4}$ Liquid chromatography-mass spectrometry assays are highly specific, sensitive, and precise and can detect hormones found in low concentrations, with a higher and more accurate correlation coefficient between free thyroxine and the log-transformed thyrotropin level than that obtained on immunoassays. ${ }^{5}$ The use of such assays can avoid the misclassification of thyroid disorders, which could have an effect on the care of patients and on research.

\author{
Fady Hannah-Shmouni, M.D. \\ National Institutes of Health \\ Bethesda, MD \\ fady.hannah-shmouni@nih.gov \\ Steven J. Soldin, Ph.D. \\ National Institutes of Health Clinical Center \\ Bethesda, MD \\ No potential conflict of interest relevant to this letter was re- \\ ported.
}

1. Baloch Z, Carayon P, Conte-Devolx B, et al. Laboratory medicine practice guidelines: laboratory support for the diagnosis and monitoring of thyroid disease. Thyroid 2003;13:3-126.

2. Toldy E, Locsei Z, Szabolcs I, Bezzegh A, Kovács GL. Protein interference in thyroid assays: an in vitro study with in vivo consequences. Clin Chim Acta 2005;352:93-104.

3. Welsh KJ, Soldin SJ. Diagnosis of endocrine disease: how reliable are free thyroid and total T3 hormone assays? Eur J Endocrinol 2016;175:R255-R263.

4. Gounden V, Jonklaas J, Soldin SJ. A pilot study: subclinical hypothyroidism and free thyroid hormone measurement by immunoassay and mass spectrometry. Clin Chim Acta 2014;430: 121-4.

5. Jonklaas J, Kahric-Janicic N, Soldin OP, Soldin SJ. Correlations of free thyroid hormones measured by tandem mass spectrometry and immunoassay with thyroid-stimulating hormone across 4 patient populations. Clin Chem 2009;55:1380-8.

DOI: 10.1056/NEJMc1709989

TO THE EDITOR: Stott et al. conclude that levothyroxine provided no apparent benefits in older persons with subclinical hypothyroidism. However, even high-normal levels of thyrotropin ( $>2.5$ mIU per liter) are associated with metabolic risk factors. ${ }^{1}$ Moreover, geriatric patients are typically taking multiple drugs. The intake of levothyroxine too close to the consumption of food or drink can cause relevant fluctuations in the results on thyroid-function testing. ${ }^{2,3}$ Such fluctuations can result in on-and-off overreplacement, with clinical symptoms of thyrotoxicosis that may have obscured the beneficial effects of levothyroxine replacement. The trial protocol mentions that levothyroxine was prescribed to be taken "before breakfast" without further specification of the precise timing of administration. We have implemented an update in the recent versions of the British National Formula suggesting that levothyroxine should be taken at least 30 minutes before breakfast and the intake of caffeine-containing beverages to facilitate more reliable absorption, a recommendation that is in line with those of other countries, including the United States. ${ }^{4}$ Outcomes of the trial by Stott et al. might have been different if the investigators had made more specific recommendations regarding the precise timing of levothyroxine intake, with possibly important implications for the treatment 
of geriatric patients with subclinical hypothyroidism.

\section{Martin O. Weickert, M.D.}

University Hospitals Coventry and Warwickshire NHS Trust Coventry, United Kingdom

martin.weickert@uhcw.nhs.uk

Ioannis Kyrou, M.D., Ph.D.

Aston Medical School

Birmingham, United Kingdom

No potential conflict of interest relevant to this letter was reported.

1. Ruhla S, Weickert MO, Arafat AM, et al. A high normal TSH is associated with the metabolic syndrome. Clin Endocrinol (Oxf) 2010;72:696-701.

2. Bach-Huynh TG, Nayak B, Loh J, Soldin S, Jonklaas J. Timing of levothyroxine administration affects serum thyrotropin concentration. J Clin Endocrinol Metab 2009;94:3905-12.

3. Benvenga S, Bartolone L, Pappalardo MA, et al. Altered intestinal absorption of L-thyroxine caused by coffee. Thyroid 2008; 18:293-301.

4. Avoid food-drug interactions: a guide from the National Consumers League and U.S. Food and Drug Administration. Silver Spring, MD: Food and Drug Administration (https://www .fda.gov/downloads/drugs/resourcesforyou/consumers/buyingusing medicinesafely/ensuringsafeuseofmedicine/generaluseofmedicine/ ucm229033.pdf).

DOI: 10.1056/NEJMc1709989

THE AUTHORS REPLY: Ogunsakin et al. ask for post-treatment free thyroxine values. Although we did not measure such levels routinely, we did report levels in a subsample of the participants, in whom levels of free thyroxine were 2.3 pmol per liter higher in the levothyroxine group than in the placebo group (Table S2 in the Supplementary Appendix of our article, available with the full text at NEJM.org). We agree that lipid levels in our trial will be of great interest. The results of such assays will be included as part of our portfolio of work on the trial biobank.

Ogunsakin et al. note an imbalance between the levothyroxine group and the placebo group in the point estimates for cardiovascular deaths and deaths from any cause. There was 1 cardiovascular death plus 4 noncardiovascular deaths in the placebo group (during 577.9 person-years of follow-up) as compared with 2 and 8 , respectively, in the levothyroxine group (during 586.9 person-years of follow-up). The confidence intervals for the treatment effect are very wide (hazard ratio for death from any cause, 1.91; 95\% confidence interval, 0.65 to 5.60 ), so it is currently not possible to state whether levothyroxine treatment might be harmful to or indeed beneficial for these outcomes.

We agree with van Vliet et al. that our trial was underpowered to determine whether treatment of subclinical hypothyroidism with levothyroxine reduces the risk of cardiovascular disease. Mendelian randomization studies can provide useful information to help determine whether epidemiologic associations are potentially causal, and we note the finding of their research with interest.

All the measures of thyrotropin and free thyroxine in our trial were determined by means of immunoassay with the use of established methods. Although analyses of thyrotropin on immunoassay platforms are considered to be reliable, the validity of immunoassay measures of free thyroxine has been questioned. ${ }^{1}$ The hypothesis of Hannah-Shmouni and Soldin - that the use of equilibrium dialysis or ultrafiltration assays to identify patients with low free thyroxine levels might help in identifying those who would benefit from levothyroxine treatment - is interesting but remains to be proven. We hope to address this issue as part of our work on the trial biobank.

We agree with the comments of Weickert and Kyrou on the timing of levothyroxine administration and absorption. ${ }^{2}$ Our simple instructions were to take the medicine before breakfast. However, since we adjusted the dose to achieve thyrotropin levels within the reference range, the timing of the receipt of levothyroxine should not have influenced the outcomes.

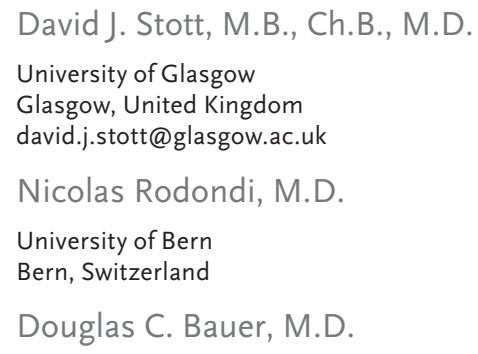

\section{for the TRUST Study Group}

Since publication of their article, the authors report no further potential conflict of interest.

1. Gounden V, Jonklaas J, Soldin SJ. A pilot study: subclinical hypothyroidism and free thyroid hormone measurement by immunoassay and mass spectrometry. Clin Chim Acta 2014;430:121-4. 2. McMillan M, Rotenberg KS, Vora $\mathrm{K}$, et al. Comorbidities, concomitant medications, and diet as factors affecting levothyroxine therapy: results of the CONTROL Surveillance Project. Drugs R D 2016;16:53-68.

DOI: $10.1056 / N E J M c 1709989$

Correspondence Copyright (๑ 2017 Massachusetts Medical Society. 